\title{
The burden of progressive fibrotic interstitial lung disease across the UK
}

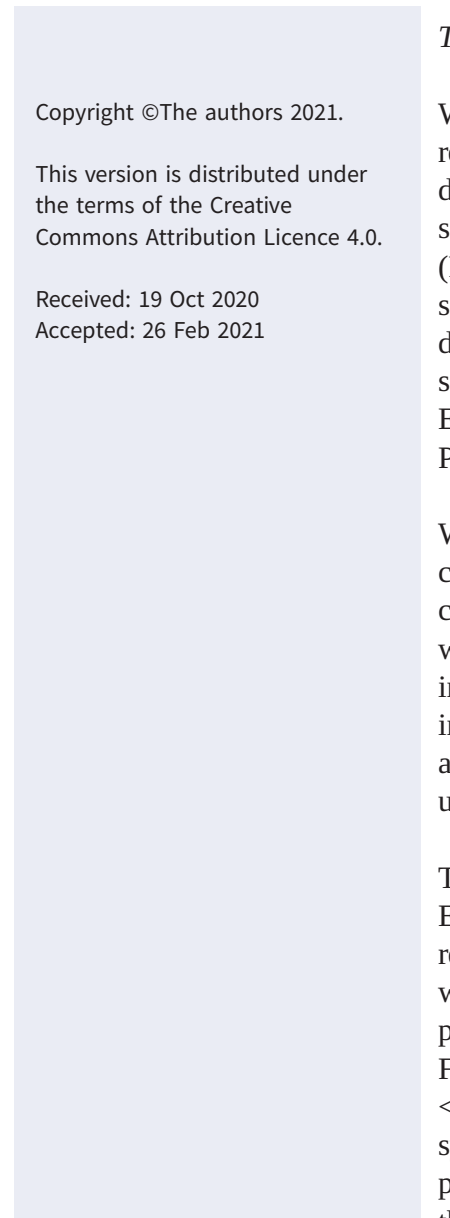

To the Editor:

While idiopathic pulmonary fibrosis (IPF) remains the exemplar progressive fibrotic lung disease, there remains a cohort of non-IPF fibrotic lung diseases (fILD) which adopt a similar clinical behaviour to IPF despite therapy [1]. This phenotypically related group of conditions, where progression of disease is similar to that seen in IPF, have recently been described as progressive fibrotic interstitial lung diseases (PF-ILD) [2]. Historically, treatments for these cases have been limited though given the phenotypic similarities many cases may have been given a multidisciplinary working diagnosis of IPF based on their disease behaviour [3]. The INBUILD trial broadened the scope of treatable fILD by demonstrating a significant benefit of Nintedanib in patients with fILD and progressive disease [4]. In response to this the European Commission approved an additional indication for nintedanib in adults for the treatment of PF-ILD in July 2020.

While research interest grows in the progressive phenotype and debates about the optimal diagnostic criteria continue the incidence of patients with PF-ILD potentially eligible for treatment according to the criteria laid out in the INBUILD trial remains unclear. Previous attempts to estimate the proportion of fILD who develop a progressive fibrotic phenotype have either used estimates based on the disease behaviour of individual conditions [5], interviews with experts [6] or analysis of insurance claims [7]. This has resulted in estimates ranging from 18 to $40 \%$ of all fILD that will develop progressive disease. With the anticipated approval of therapeutic interventions for this cohort of patients worldwide, including in the UK, there is an urgent need to refine these estimates in a real-world population to enable appropriate service provision.

This retrospective, observational study therefore aimed to estimate the incidence of PF-ILD across England. Nine centres providing commissioned tertiary referral services for ILD were included. All new referrals seen for their first outpatient clinic appointment between August 1, 2017, and January 31, 2018, were assessed against the diagnostic criteria for PF-ILD laid out in the INBUILD trial [8] and, in particular, the criteria for progression: relative decline in forced vital capacity (FVC) \% predicted $\geqslant 10 \%$, or FVC decline $\geqslant 5 \%$ but $<10 \%$, combined with worsening respiratory symptoms, or FVC decline $\geqslant 5 \%$ but $<10 \%$, combined with radiological progression; or radiological progression with worsening respiratory symptoms. A full chart and imaging review was undertaken of all the subjects. Continuous variables are presented as mean \pm SD, and categorical variables as proportions. Time-to-event curves were calculated using the Kaplan-Meier method and compared with the use of the log-rank test.

A total of 2368 patients with ILD were assessed across the nine centres. 619 patients were diagnosed and managed as IPF and therefore excluded, leaving 1749 patients with fILD who were screened against the INBUILD criteria for progression, to identify cases of PF-ILD either at the first clinical review, or in the subsequent 2 years of follow up. In the cohort of patients at risk of developing PF-ILD the INBUILD criteria were met in 14.5\% (253 out of 1749) of all new non-IPF fILD referrals despite standard therapy, with a range between these specialist ILD centres from $8.9 \%$ to $23.6 \%$ of total cases. The average time from referral to specialist centre to diagnosis of progressive phenotype was $311 \pm 273$ days and, at the time of referral, 20\% of patients demonstrated progressive disease (66 out of 253) despite standard therapy. Almost all patients received at least one immunosuppressive agent, with the majority receiving either oral or intravenous corticosteroids (96\%). A number of second-line agents were employed with mycophenolate

\section{Shareable abstract (@ERSpublications)}

Almost $15 \%$ of new referrals with non-IPF fibrotic ILD go on to develop a progressive fibrotic phenotype and would benefit from antifibrotic therapy https://bit.ly/3uPhCIN

Cite this article as: Simpson T, Barratt SL, Beirne P, et al. The burden of progressive fibrotic interstitial lung disease across the UK. Eur Respir J 2021; 58: 2100221 [DOI: 10.1183/13993003.00221-2021]. 
(46\%) the most commonly used. Five of the subjects with PF-ILD received antifibrotic therapy on compassionate grounds.

The most common diagnoses associated with a PF-ILD phenotype were chronic hypersensitivity pneumonitis (84 (33.2\%) out of 253), unclassifiable ILD (44 (17.3\%) out of 253), connective tissue disease-associated ILDs including rheumatoid arthritis-associated ILD (42 (16.6\%) out of 253) and non-specific interstitial pneumonitis (36 (14.2\%) out of 253). In the PF-ILD cases, the mean age was $68 \pm 12.4$ years and, interestingly, $53.4 \%$ of the cohort was female, compared with the well-recognised male predominance seen in IPF. This is likely driven by the significant female predominance in both chronic hypersensitivity pneumonitis and connective tissue disease which make up almost half of the PF-ILD cases.

Patients with progressive disease had a significantly higher mortality compared to those with non-progressive fILD (hazard ratio, 3.32; 95\% confidence interval, 2.53-4.37; $\mathrm{p}=<2 \times 10^{-16}$ ). Indeed, the survival of patients with PF-ILD was no different to the subjects with IPF (hazard ratio, 1.06; 95\% CI 0.84-1.35; $\mathrm{p}=0.6$ ) (figure 1).

Of the progression events, the majority were driven by a measured drop in FVC, with more than half of patients $(52.2 \%)$ experiencing a drop of $\geqslant 10 \%$. A further quarter of patients $(24.1 \%)$ were diagnosed with progressive disease on the basis of radiological and symptomatic progression alone without a spirometric deterioration. The remainder experienced a decline of FVC between 5 and 10\% with either radiological (15.8\%) or symptomatic (7.9\%) progression.

The variations between centres and clinicians in diagnostic pathways, approaches to follow-up and definitions of progression has previously made it difficult to define and assess this cohort of patients. One of the strengths of our approach was the central collation and uniform application of the INBUILD inclusion criteria. However, this was done retrospectively and this is the main limitation of our study. While the INBUILD trial criteria are mostly objectively measurable phenomena, the definition of progressive symptoms may allow some biasing towards inclusion in those cases where spirometric progression was either not evidenced or not available, thus increasing the numbers of cases. Over a quarter of referrals received a final multidisciplinary team diagnosis of IPF, and this is often pragmatic and based on their clinical disease behaviour, to allow access to antifibrotic therapy. However, a patient's initial clinical and radiological features may have had more in keeping with a different ILD but with a PF-ILD phenotype. While all of the cases underwent local ILD multidisciplinary assessment, we did not undertake any central reassessment and therefore some cases of non-IPF PF-ILD may have been missed. Progression was only assessed at presentation and over a period of 2 years, as per the INBUILD screening criteria; however, we do know that progression may occur later during follow-up [9] and therefore some late

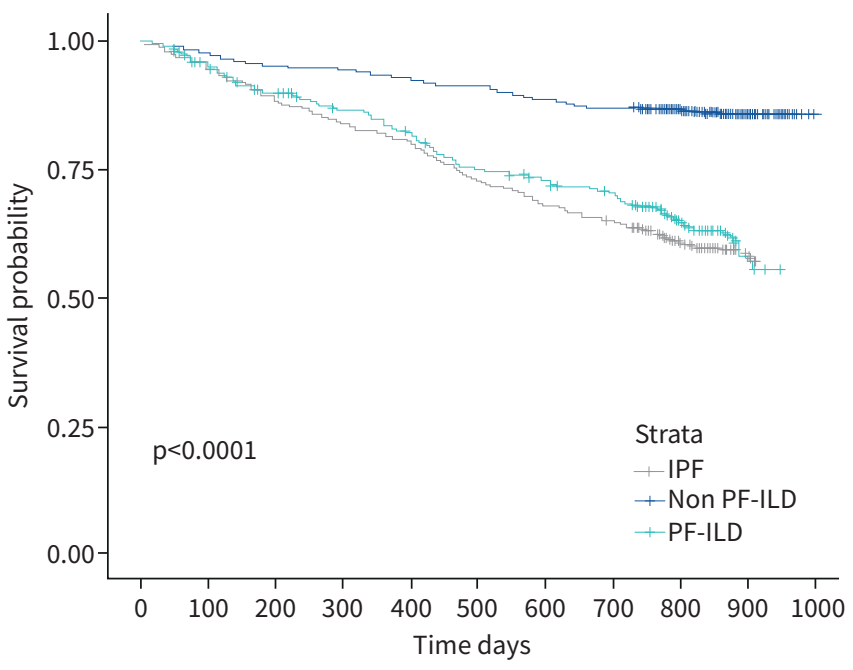

FIGURE 1 Kaplan-Meier curves comparing survival between patients with idiopathic pulmonary fibrosis (IPF), progressive fibrotic interstitial lung diseases (PF-ILD) and non-progressive non-IPF fibrotic lung diseases (non PF-ILD). Log-rank $p$ test value is reported. 
progressors would not have been captured in this analysis. Our estimates therefore may be if anything an underestimate but importantly they reflect current clinical practice, which we aimed to capture.

This study represents a fair and balanced approach to assessing the incidence of objectively measurable and treatable PF-ILDs in the UK. A rate of $14.5 \%$ of new referrals with non-IPF ILD is less than that reported in previous studies however our methodology is likely to give a more accurate result than estimates based on extrapolation from general disease statistics, from physician-reported estimates prone to significant biases, or insurance claim processes also substantially prone to bias. This information has implication for workforce planning and the funding of anti-fibrotic therapy in the UK and beyond.

Thomas Simpson ${ }^{1}$, Shaney L. Barratt $\circledast^{2,3}$, Paul Beirne ${ }^{4}$, Nazia Chaudhuri ${ }^{5}$, Anjali Crawshaw ${ }^{6}$, Louise E. Crowley ${ }^{6}$, Sophie Fletcher ${ }^{7}$, Michael A. Gibbons ${ }^{8}$, Philippa Hallchurch ${ }^{2}$, Laura Horgan ${ }^{4}$, leva Jakaityte ${ }^{8}$, Thomas Lewis ${ }^{2}$, Tom McLellan ${ }^{9}$, Katherine J. Myall ${ }^{1}$, Ryan Miller ${ }^{8}$, David J.F. Smith ${ }^{10}$, Stefan Stanel ${ }^{5}$, Muhunthan Thillai $\odot^{9}$, Fiona Thompson ${ }^{7}$, Timothy Wallis ${ }^{7}$, Zhe Wu $\oplus^{10,11}$, Philip L. Molyneaux $\oplus^{10,11,12}$ and Alex G. West $\oplus^{1,12}$

${ }^{1}$ Dept of Respiratory Medicine, Guy's and St Thomas' NHS Foundation Trust, London, UK. ${ }^{2}$ Bristol Interstitial Lung Disease Service, North Bristol NHS trust, Southmead Hospital, Bristol, UK. ${ }^{3}$ Academic Respiratory Unit, University of Bristol, Bristol, UK. ' Leeds Interstitial Lung Disease Service, St James's University Hospital, Leeds, UK. ${ }^{5}$ North West Lung Centre, Wythenshawe Hospital, Manchester, UK. ${ }^{6}$ Birmingham Interstitial Lung Disease Unit, Queen Elizabeth Hospital Birmingham, University Hospitals Birmingham NHS Foundation Trust, Birmingham, UK. ${ }^{7}$ University Hospitals Southampton NHS Foundation Trust, Southampton, UK. ${ }^{8}$ South West Peninsula ILD Network, Royal Devon and Exeter Foundation NHS Trust, Exeter, UK. ${ }^{9}$ Dept of Interstitial Lung Disease, Royal Papworth Hospital NHS Foundation Trust, Cambridge, UK. ${ }^{10}$ Royal Brompton Hospital, London, UK. ${ }^{11}$ National Heart and Lung Institute, Imperial College London, London, UK. ${ }^{12}$ Contributed equally as last authors.

Corresponding author: Philip L. Molyneaux (p.molyneaux@imperial.ac.uk)

Conflict of interest: T. Simpson reports non-financial support (educational support/leadership course) from Boehringer Ingelheim, outside the submitted work. S.L. Barratt reports grants, personal fees and non-financial support for meeting attendance from Boehringer Ingelheim, outside the submitted work. P. Beirne reports personal fees for consultancy and non-financial support for meeting attendance from Boehringer Ingelheim Pharmaceuticals, outside the submitted work. N. Chaudhuri reports grants and personal fees for consultancy from Boehringer Ingelheim, personal fees for consultancy from Roche, outside the submitted work. A. Crawshaw has received honoraria from Boehringer Ingelheim for educational meetings (chair/talks) and grants for virtual attendance at international conferences. L.E. Crowley has nothing to disclose. S. Fletcher has nothing to disclose. M.A. Gibbons has served on advisory boards and received support to attend conferences from Roche and Boehringer Ingelheim. P. Hallchurch has nothing to disclose. L. Horgan has nothing to disclose. I. Jakaityte has nothing to disclose. T. Lewis has nothing to disclose. T. McLellan reports unrestricted educational grants from Boehringer Ingelheim, outside the submitted work. K.J. Myall has nothing to disclose. R. Miller has nothing to disclose. D.J.F. Smith has nothing to disclose. S. Stanel has nothing to disclose. M. Thillai has nothing to disclose. F. Thompson has nothing to disclose. T. Wallis has nothing to disclose. Z. Wu has nothing to disclose. P.L. Molyneaux, via his institution, received industry-academic funding from AstraZeneca and has received speaker and consultancy fees from Boehringer Ingelheim and Hoffman-La Roche, outside the submitted work. A.G. West has nothing to disclose.

Support statement: P.L. Molyneaux is supported by an Action for Pulmonary Fibrosis Mike Bray fellowship.

\section{References}

1 Brown KK, Martinez FJ, Walsh SLF, et al. The natural history of progressive fibrosing interstitial lung diseases. Eur Respir J 2020; 55: 2000085.

2 Cottin V, Hirani NA, Hotchkin DL, et al. Presentation, diagnosis and clinical course of the spectrum of progressive-fibrosing interstitial lung diseases. Eur Respir Rev 2018; 27: 180076.

3 Cottin V. Treatment of progressive fibrosing interstitial lung diseases: a milestone in the management of interstitial lung diseases. Eur Respir Rev 2019; 28: 190109.

4 Flaherty KR, Wells AU, Cottin V, et al. Nintedanib in progressive fibrosing interstitial lung diseases. $N$ Engl J Med 2019; 381: 1718-1727. 
5 Olson AL, Gifford AH, Inase N, et al. The epidemiology of idiopathic pulmonary fibrosis and interstitial lung diseases at risk of a progressive-fibrosing phenotype. Eur Respir Rev 2018; 27: 180077.

6 Wijsenbeek M, Kreuter M, Olson A, et al. Progressive fibrosing interstitial lung diseases: current practice in diagnosis and management. Curr Med Res Opin 2019; 35: 2015-2024.

7 Olson AL, Maher TM, Acciai V, et al. Healthcare resources utilization and costs of patients with Non-IPF progressive fibrosing interstitial lung disease based on insurance claims in the USA. Adv Ther 2020; 37: 3292-3298.

8 Flaherty KR, Brown KK, Wells AU, et al. Design of the PF-ILD trial: a double-blind, randomised, placebo-controlled phase III trial of nintedanib in patients with progressive fibrosing interstitial lung disease. BMJ Open Respir Res 2017; 4: e000212.

9 Nasser M, Larrieu S, Si-Mohamed S, et al. Progressive fibrosing interstitial lung disease: a clinical cohort (the PROGRESS ${ }^{\circledR}$ study). Eur Respir J 2020; 57: 2002718. 